\title{
A novel $p$-harmonic descent approach applied to fluid dynamic shape optimization
}

\author{
Peter Marvin Müller ${ }^{1} \mathbb{0} \cdot$ Niklas Kühl $^{1} \cdot$ Martin Siebenborn $^{2} \cdot$ Klaus Deckelnick $^{3} \cdot$ Michael Hinze $^{4} \cdot$ Thomas Rung $^{1}$
}

Received: 30 March 2021 / Revised: 4 July 2021 / Accepted: 23 July 2021 / Published online: 26 August 2021

(c) The Author(s) 2021

\begin{abstract}
We introduce a novel method for the implementation of shape optimization for non-parameterized shapes in fluid dynamics applications, where we propose to use the shape derivative to determine deformation fields with the help of the $p$ - Laplacian for $p>2$. This approach is closely related to the computation of steepest descent directions of the shape functional in the $W^{1, \infty}$ - topology and refers to the recent publication Deckelnick et al. (A novel $W^{1, \infty}$ approach to shape optimisation with Lipschitz domains, 2021), where this idea is proposed. Our approach is demonstrated for shape optimization related to drag-minimal free floating bodies. The method is validated against existing approaches with respect to convergence of the optimization algorithm, the obtained shape, and regarding the quality of the computational grid after large deformations. Our numerical results strongly indicate that shape optimization related to the $W^{1, \infty}$-topology-though numerically more demanding-seems to be superior over the classical approaches invoking Hilbert space methods, concerning the convergence, the obtained shapes and the mesh quality after large deformations, in particular when the optimal shape features sharp corners.
\end{abstract}

Keywords Shape optimization $\cdot W^{1, \infty}$-steepest descent $\cdot p$-Laplace relaxation $\cdot$ Adjoint optimization

\section{Introduction}

Adjoint-based local optimization has been matured toward an efficient industrially applied strategy, e.g., Othmer (2014), Papoutsis-Kiachagias and Giannakoglou (2016). When attention is given to shape optimization, the aim is to find optimal shapes regarding a physical quantity $J$, e.g., the drag force experienced by an obstacle. Mathematically speaking, a shape functional $J$ is minimized subject

Responsible Editor: Nathalie Bartoli

Peter Marvin Müller

peter.marvin.mueller@tuhh.de

1 Institute for Fluid Dynamics and Ship Theory, Hamburg University of Technology, Am Schwarzenberg-Campus 4, 21073 Hamburg, Germany

2 Department of Mathematics, Hamburg University, Bundesstrasse 55, 20146 Hamburg, Germany

3 Department of Mathematics, Otto-von-Guericke-Universität Magdeburg, Universitätsplatz 2, 39106 Magdeburg, Germany

4 Mathematisches Institut, Universität Koblenz-Landau, Campus Koblenz, Universitätsstraße 1, 56070 Koblenz, Germany to partial differential equation (PDE) constraints. The latter typically govern the physics, e.g., the conservation of mass and momentum. A crucial part of the shape optimization procedure is the choice of the descent direction that provides an update rule for the design variable. In our case, the design variable refers to the underlying geometry $\Omega \subset \mathbb{R}^{d}$ which is a subset of the $d$-dimensional Euclidean space, the boundary $\partial \Omega$ or parts of the boundary $\Gamma$. The descent direction is usually employed to converge a sequence of shape updates via a deformation field $\mathbf{u}$. First attempts used the directional derivative $J^{\prime}$, also referred to as shape derivative in order to formulate a shape update rule (Sokolowski and Zolésio 1992; Kawohl et al. 1998; Delfour and Zolésio 2011). Because shapes are not elements of a vector space, e.g., there is no meaningful definition of the summation of two shapes, the shape derivative is defined by introducing deformations that make shapes variable and thus allows the definition of directional derivatives. With the help of shape calculus, cf. Sokolowski and Zolésio (1992) and Delfour and Zolésio (2011) for a mathematical perspective or Schmidt et al. (2013) and Kühl et al. (2019) for an engineering perspective, a shape derivative can be computed that relates to a scalar field $\gamma$ defined on the boundary $\Gamma$. We want to refer to $\gamma$ as the local shape sensitivity. Mind that the computation of 
the shape derivative with shape calculus is a rather involved task and heavily depends on the objective functional $J$, the PDE constraints that apply as well as the domain $\Omega$ and the boundary $\partial \Omega$, respectively. In Pironneau (1973), the shape is updated using the local shape sensitivity $\gamma$ to perform the deformation in normal direction $\mathbf{n}$ of the boundary $\Gamma$. Using the descent direction $\mathbf{u}=-\gamma \mathbf{n}$ yields a contribution $\mathbf{u} \cdot \mathbf{n}=-\gamma$ to reducing the objective functional, and is a popular approach within shape optimization, cf. Vassberg and Jameson (2006, 2006). The attempt is limited since it often yields shapes with rough/noisy boundaries (Stück and Rung 2011; Kröger and Rung 2015) and distorted near-wall meshes which in turn hamper the preservation of numerical accuracy during the optimization procedure (Stavropoulou et al. 2014; Bletzinger 2014). In fluid dynamics and neighboring applications, the computational grid is frequently morphed and not renewed after each optimization step. Thereupon, attempts follow in order to gain higher regularity of the deformation. One approach is based on the definition of a shape gradient grad $J$ by an inner product and the shape derivative $J^{\prime}$. Here the shape gradient is identified by the Riesz representation of the directional derivative of the shape functional. Even though this leads to smoother deformations, the approach is algorithmic challenging due to solving a PDE on a hyperplane, and also mathematically questionable in the general case, see also Allaire et al. (2021) and cf. Sect. 2. In this regard, different gradients are associated with different transformations applied to the shape derivative, and several techniques have been proposed to increase the regularity of the shape updates:

(a) CAD-related shape definitions connect the node-based shape derivatives to the CAD parameterization using the chain rule of differentiation, cf. Löhner et al. (2003) and Robinson et al. (2012). The procedure couples the various local derivatives and thereby ensures smooth shapes. However, the rigid finite dimensional initial CAD parameterization limits the attainable shapes and different CAD models may result in different optimal shapes.

(b) A coupling of mesh node updates using either local shape functions, e.g., FE-type functions Soto et al. (2002, 2004), or global shape functions, e.g., HicksHenne approaches Hicks and Henne (1978).

(c) A more rigor approach of Jameson and Vassberg (2005) and Vassberg and Jameson (2006a, b) applies an implicit, continuous smoothing operator to either the shape derivative $J^{\prime}$ or the deformation field $\mathbf{u}$, based on an extended definition of the inner product, frequently labeled 'Sobolev-gradient'. Applying the smoothing operation on the surface leads to the Laplace-Beltrami operator and a related surface metric. For computational reasons, the practice is often performed in an explicit manner, cf. Bletzinger (2014). The explicitly filtered local shape sensitivity, e.g., using consistent kernel functions Kröger and Rung (2015), marks a firstorder approximation to the implicit Sobolev-gradient Stück and Rung (2011).

All strategies $(\mathrm{a}-\mathrm{c})$ essentially couple node updates and thereby obtain smooth design updates. Having updated the discrete design surface, the subsequent numerical investigation of the updated design also requires an update of the computational mesh, i.e., the shape gradient of the design surface needs to be extended into the domain. The habitat of the shape gradient depends on the surface metric and can be surface as well as volume-based. Prominent examples refer to above-mentioned Laplace-Beltrami (LB) or the Steklov-Poincaré (SP) metric, c.f. Schulz and Siebenborn (2016). The first approach (LB) exclusively operates in the tangent space of the design surface, the latter (SP) leads to a domain formulation, where results are subsequently projected on the controlled shape. The SP strategy gives the shape update of the design surface and mesh using the shape sensitivity (Schulz and Siebenborn 2016; Haubner et al. 2020; Allaire et al. 2021). A similar approach has been used in Azegami and Wu (1996) and Azegami et al. (2013) were the descent direction is obtained by the solution of a linear elastic-like problem with the negative shape derivative as Neumann data. The approach is referred to as $H^{1}$ gradient method or traction method because the Neumann condition can be interpreted as a fictional traction. The volume-based SP approach is particularly attractive for optimization procedures which prefer mesh morphing over re-meshing strategies, as it is customary for engineering simulations. While re-meshing can be automated, the lack of fair restart capabilities becomes prohibitively expensive in practical applications. Moreover, the use of standardized, HPC capable solution routines supplied by the flow solver (assembling, solving, etc.) represent another significant benefit of the SP approach.

A different avenue is taken by the phase field method for fluid mechanic shape optimization, where the shape of the sought domain is approximated by the zero level set of a phase field function. This turns the shape optimization problem into a PDE constrained optimization problem where the phase field enters as control in the coefficients of the PDE. This allows to apply the complete algorithmic machinery of PDE constrained optimization methods to this formulation of the fluid dynamic shape optimization problems, and this approach also naturally allows topology changes of the shape. However, numerical methods also for this approach encounter problems in situations where the sought shape needs to develop kinks and/or corners. This approach is proposed in Borrvall and Petersson (2003), and in a couple of 
papers investigated for hydrodynamic shape optimization problems Garcke et al. (2015, 2016, 2018).

Although smooth shapes may be desirable for different reasons, they are not necessarily optimal. If the optimum involves a kink or a corner, the above-mentioned strategies to obtain smooth shape updates display difficulties to capture such optima from curved initial configurations, if the respective region is (initially) not resolved by very fine grids. Section 4 of the present paper discusses a classical example of a pointed optimal shape. The same is true for counterpart situations, i.e. to transform an initially kinked shape into a curved optimum. Though this might be possible, the convergence is often fairly slow.

The present study aims to convey the merits of an alternative strategy to compute the shape deformation from shape derivatives in the context of CAD-free-aka nodebased-shape optimization. The $p$-Laplace operator is used in a volume-based formulation along the route of the SP metric. The approach is industrially feasible and supports unstructured meshes. Applications refer to $2 D$ and $3 D$ fluid dynamic shape optimization ranging from laminar to turbulent external flows around free floating objects with fixed displacement.

The remainder of the paper is structured as follows: Sect. 2 outlines the mathematical framework and the rationale that leads to the $p$-Laplace problem to approximate the steepest descent direction within the $W^{1, \infty}$ - topology together with a discussion of our fluid dynamic shape optimization problem. Section 3 presents the solution algorithm. Section 4 applies the approach to three test cases and the manuscript closes with conclusions in Sect. 5.

\section{Mathematical framework}

In this section, we want to outline the basic idea behind the $p$-harmonic approach and briefly recall the concept of shape optimization.

Fig. 1 Schematic sketch of the computational domain
For this purpose let $J: \mathcal{A} \rightarrow \mathbb{R}$ denote a shape functional, where $\mathcal{A}$ denotes the set of admissible domains which has to be specified in the respective application. In our setting, the set $\mathcal{A}$ is specified through Fig. 1, and the shape functional with (5) is given in (6a). For the algorithmic minimization of $J$ for a given domain $\Omega \in \mathcal{A}$ we intend to specify descent vector fields $\mathbf{u}^{*}: \mathbb{R}^{d} \rightarrow \mathbb{R}^{d}$ such that $J^{\prime}\left(\Omega ; \mathbf{u}^{*}\right)<0$ holds, where $J^{\prime}\left(\Omega ; \mathbf{u}^{*}\right)$ denotes the shape derivative of $J$ at $\Omega$ in direction of $\mathbf{u}^{*}$. The perturbed domain $\tilde{\Omega}$ then has the form

$\tilde{\Omega}=\mathbf{T}_{t}(\Omega):=\left(\mathbf{i d}+t \mathbf{u}^{*}\right)(\Omega)$,

where $t>0$ is a step size specified in the respective minimization algorithm and id is the identity operator. However, descent in this context requires to specify an appropriate topology. Moreover, it frequently is required that Lipschitz domains $\Omega$ are mapped to Lipschitz domains $\tilde{\Omega}$. Common practical approaches use Hilbert Space methods, i.e., seek descent vector fields $\mathbf{u}^{*}$ determined with the help of the shape derivative by

$a\left(\mathbf{u}^{*}, \mathbf{w}\right)=J^{\prime}(\Omega ; \mathbf{w})$ for all $\mathbf{w} \in H$,

where $(H, a(\cdot, \cdot))$ denotes an appropriate Hilbert space, see e.g., Allaire et al. (2021, Sect. 5-9) for an extensive discussion of approaches related to the Hilbert space setting. This requires to compute the Riesz representative of the functional $J^{\prime}(\Omega ; \cdot)$. A typical choice of $H$ is $H^{m}\left(\Omega, \mathbb{R}^{d}\right)$, where however $m \in \mathbb{N}$ has to be chosen large enough to obtain a Lipschitz transformation. A way around this would be to directly choose $\mathbf{u}^{*} \in W^{1, \infty}\left(\Omega, \mathbb{R}^{d}\right)$ as a direction of steepest descent for $J$ at $\Omega$, where $W^{1, \infty}\left(\Omega, \mathbb{R}^{d}\right)$ denotes the set of Lipschitz transformations from $\Omega$ to $\mathbb{R}^{d}$. For the requirements on transformations in shape optimization we refer to Sokolowski and Zolésio (1992), Delfour and Zolésio (2011) and Allaire et al. (2021). This leads to the minimization problem

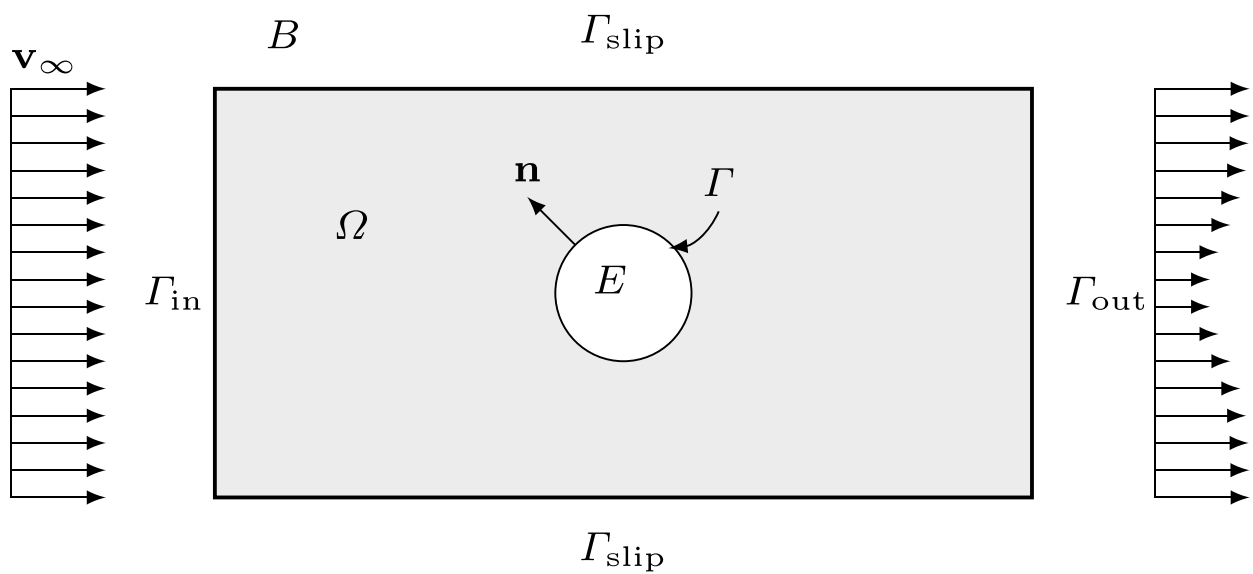


$\min _{\mathbf{u} \in W^{1, \infty}\left(\Omega, \mathbb{R}^{d}\right),\|\mathbf{u}\|_{W^{1, \infty}} \leq 1} J^{\prime}(\Omega ; \mathbf{u})$,

which is challenging both from the mathematical and the numerical perspective, since it represents a variational problem in the non-reflexive Banach space $W^{1, \infty}\left(\Omega, \mathbb{R}^{d}\right)$. Variational problems of this kind are studied in e.g., Ishii and Loreti (2005), where it is proposed to approach solutions $\mathbf{u}^{*}$ of problem (3) by a sequence of solutions $\mathbf{u}_{p}^{*} \in W^{1, p}\left(\Omega, \mathbb{R}^{d}\right)$ of the variational problem

$\min _{u_{p} \in W^{1, p}\left(\Omega, \mathbb{R}^{d}\right)} \frac{1}{p} \int_{\Omega}\left(\nabla \mathbf{u}_{p}: \nabla \mathbf{u}_{p}\right)^{\frac{p}{2}} \mathrm{~d} x+J^{\prime}\left(\Omega ; \mathbf{u}_{p}\right)$

for $p>2$, compare (Ishii and Loreti 2005, Propositions $5.2,5.3)$ for the mathematical analysis of the limit process $p^{*} \rightarrow \infty$. Note that (4) defines a strictly convex functional in $\mathbf{u}_{p}$ for $p>1$. For the special case $p=2$ we recover a Hilbert space setting as described above, and refer to problem (4) as $p$-Laplace relaxation of problem (3). In the next section, we adapt problem (4) to our fluid dynamic setting and use its solutions as descent directions in our augmented Lagrange algorithm for the numerical solution of our shape optimization problem.

\subsection{Optimization problem}

We now introduce the mathematical setting for our hydrodynamic shape optimization problem, where we refer to Fig. 1 for the geometrical setting and the notation. The governing equations here are either given by the stationary Navier-Stokes equations for incompressible fluids and laminar flows or the Reynolds averaged Navier-Stokes (RANS) equations which we consider for turbulent flow at high Reynolds numbers. Note that in the latter case turbulence modeling is required. Our aim is to find the shape of a generic obstacle $E \subset B$ with Lipschitz boundary located within the flow channel $B \subset \mathbb{R}^{d}$ which has minimal drag. Thus, $\Omega=B \backslash E$, as shown in Fig. 1. The state then is given by the velocity $\mathbf{v}: \Omega \rightarrow \mathbb{R}^{d}$ and pressure $p: \Omega \rightarrow \mathbb{R}$, which are assumed to be unique on $\Omega$, and thus a mapping $\Omega \mapsto \mathbf{y}(\Omega)=(\mathbf{v}, p)$ exists.

It is considered that the domain $\Omega \subset B$ is completely filled with fluid which flows in from the left boundary $\Gamma_{\text {in }}$ with a prescribed velocity $\mathbf{v}_{\infty} \in \mathbb{R}^{d}$ toward the outflow boundary $\Gamma_{\text {out }}$ on the right. The top and bottom boundaries $\Gamma_{\text {slip }}$ are considered as frictionless slip wall boundaries. For all test cases, the whole boundary $\Gamma$ of the obstacle $E$ is considered to be the control of the total force acting on its boundary, viz.

$\mathbf{F}(\Omega)=\int_{\Gamma \subset \partial \Omega}\left(\mu\left(\nabla \mathbf{v}+\nabla \mathbf{v}^{T}\right)-\mathbf{I} p\right) \cdot \mathbf{n} \mathrm{d} s$, where $\mathbf{I}$ is the identity tensor. The drag is the component of the force $\mathbf{F}(\Omega)$ in direction of the approaching flow $-\mathbf{v}_{\infty} /\left\|\mathbf{v}_{\infty}\right\|$. Thus the drag is given by the inner product $-\mathbf{v}_{\infty} /\left\|\mathbf{v}_{\infty}\right\| \cdot \mathbf{F}(\Omega)$. However, additionally to the flow state also geometric constraints need to be considered. On the one hand, the geometry has to be held in place to prevent the body from moving during the optimization process. On the other hand the geometry would shrink to a single point if the volume is not preserved. Thus, in the problem formulation the location of the barycenter and the wetted volume $\Omega$ are prescribed to be the vector $\overline{\mathbf{b}} \in \mathbb{R}^{d}$ and the constant $\bar{c}>0$, respectively. Both geometrical constraints supplement the PDE constraints and the optimization problem reads

$\min _{\Omega}-\frac{\mathbf{v}_{\infty}}{\left\|\mathbf{v}_{\infty}\right\|} \cdot \mathbf{F}(\Omega)$

subjected to

$\rho(\mathbf{v} \cdot \nabla) \mathbf{v}-\operatorname{div}\left(\mu\left(\nabla \mathbf{v}+\nabla \mathbf{v}^{T}\right)\right)=-\nabla p \quad$ in $\Omega$,

$-\nabla \cdot \mathbf{v}=0 \quad$ in $\Omega$

$\mathbf{v}=\mathbf{0} \quad$ on $\Gamma$,

$\mathbf{v}=\mathbf{v}_{\infty} \quad$ on $\Gamma_{\text {in }}$

$\mathbf{v} \cdot \mathbf{n}=0, \mathbf{n} \cdot \boldsymbol{\tau} \cdot \mathbf{t}=\mathbf{0} \quad$ on $\Gamma_{\text {slip }}$

$\mu\left(\nabla \mathbf{v}+\nabla \mathbf{v}^{T}\right) \cdot \mathbf{n}=p \mathbf{n} \quad$ on $\Gamma_{\text {out }}$,

$\mathbf{b}=\frac{\int_{\Omega} \mathbf{x} \mathrm{d} x}{\int_{\Omega} 1 \mathrm{~d} x}-\overline{\mathbf{b}}=\mathbf{0}$,

$c=\int_{\Omega} 1 \mathrm{~d} x-\bar{c}=0$,

where the local tangent direction is defined by $\mathbf{t}=\mathbf{t}_{\tau} /\left\|\mathbf{t}_{\tau}\right\|_{2}$ with the tangent projection of the shear force $\mathbf{t}_{\tau}:=\boldsymbol{\tau} \cdot \mathbf{n}-\left(\mathbf{n}^{T} \boldsymbol{\tau} \mathbf{n}\right) \mathbf{n}$ and $\boldsymbol{\tau}=\left(\mu\left(\nabla \mathbf{v}+\nabla \mathbf{v}^{T}\right)-\mathbf{I} p\right)$, and $\rho, \mu \in \mathbb{R}_{+}$are the density and molecular viscosity of the fluid. The vector containing the geometrical constraints from (6h)-(6i) is given by $\mathbf{g}=(\mathbf{b}, c)^{T}$, where $\mathbf{b}$ and $c$ refer to barycenter and volume residuals, respectively. To deal with the constraint problem above, a common approach is to introduce Lagrange multipliers. Therefore, we obtain the augmented Lagrangian of problem (6a)-(6i) as 


$$
\begin{aligned}
\mathcal{L}\left(\Omega, \mathbf{v}, p, \hat{\mathbf{v}}, \hat{p}, \lambda, \lambda_{b}, \lambda_{c}\right)= & -\frac{\mathbf{v}_{\infty}}{\left\|\mathbf{v}_{\infty}\right\|} \cdot \mathbf{F}(\Omega) \\
& +\int_{\Omega} \hat{\mathbf{v}} \cdot\left(\rho(\mathbf{v} \cdot \nabla) \mathbf{v}-\operatorname{div}\left(\mu\left(\nabla \mathbf{v}+\nabla \mathbf{v}^{T}\right)\right)+\nabla p\right) \mathrm{d} x \\
& -\int_{\Omega} \hat{p}(\nabla \cdot \mathbf{v}) \mathrm{d} x+\int_{\Gamma} \lambda \cdot \mathbf{v} \mathrm{d} s \\
& +\lambda_{b} \cdot \mathbf{b}+\lambda_{c} c+\frac{\rho_{b}}{2}\|\mathbf{b}\|^{2}+\frac{\rho_{c}}{2} c^{2},
\end{aligned}
$$

where $\rho_{b}, \rho_{c} \in \mathbb{R}_{+}$are sufficiently large penalty factors. The variables $(\hat{\mathbf{v}}, \hat{p})$ are the Lagrange multipliers for the PDE constraints $(6 \mathrm{~b})$ and $(6 \mathrm{c})$ which also are associated with the adjoint state and $\lambda \in \mathbb{R}^{d}$ is a Lagrange multiplier for considering the Dirichlet boundary condition (6d) that holds on the deformed boundary $\Gamma$. Note that when considering RANS equations the turbulence model implies the solution of additional state equations and thus corresponding Lagrange multipliers. Here we assume the influence of turbulence effects to be small which justifies the frequently employed frozen turbulence assumption, cf. Dwight and Brézillon (2006), Othmer (2008) and Stück and Rung (2013). The multipliers $\lambda_{b} \in \mathbb{R}^{d}$ and $\lambda_{c} \in \mathbb{R}$, belonging to the geometric constraints (6h) and (6i), are determined with an augmented Lagrange method and considered to be constant throughout the performance of algorithm 2, as described in detail below. Followingthe standard approach, as described in textbooks like Hinze et al. (2008), Ulbrich and Ulbrich (2012), the penalized objective function

$J(\Omega)=-\frac{\mathbf{v}_{\infty}}{\left\|\mathbf{v}_{\infty}\right\|} \cdot \mathbf{F}(\Omega)+\frac{\varrho_{b}}{2}\|\mathbf{b}\|^{2}+\frac{\varrho_{c}}{2} c^{2}$

can be expressed by the Lagrangian (7) and therewith the constraint problem (6a)-(6i) can be transformed into an unconstrained problem

$\min _{\Omega} \sup _{(\hat{\mathbf{v}}, \hat{p})} \mathcal{L}\left(\Omega, \mathbf{v}, p, \hat{\mathbf{v}}, \hat{p}, \lambda, \lambda_{b}, \lambda_{c}\right)$.

The first-order optimality system for (9) is given by the partial derivative of (7) with respect to each argument. The Fréchet derivative, w.r.t. the adjoint state $(\hat{\mathbf{v}}, \hat{p})$ gives the boundary value problem (6b)-(6g). Differentiation of (7) with respect to the state $(\mathbf{v}, p)$ and integration by parts leads to the adjoint equation system

$$
-\operatorname{div}\left(\mu\left(\nabla \hat{\mathbf{v}}+\nabla \hat{\mathbf{v}}^{T}\right)\right)-\rho(\mathbf{v} \cdot \nabla) \hat{\mathbf{v}}+\rho \nabla \mathbf{v}^{T} \hat{\mathbf{v}}=-\nabla \hat{p} \quad \text { in } \Omega,
$$

$\nabla \cdot \hat{\mathbf{v}}=0 \quad$ in $\Omega$,

$\hat{\mathbf{v}}+\frac{\mathbf{v}_{\infty}}{\left\|\mathbf{v}_{\infty}\right\|}=\mathbf{0} \quad$ on $\quad \Gamma$, $\hat{\mathbf{v}}=\mathbf{0} \quad$ on $\quad \Gamma_{\text {in }}$

$\mu\left(\nabla \hat{\mathbf{v}}+\nabla \hat{\mathbf{v}}^{T}\right) \cdot \mathbf{n}=\hat{p} \mathbf{n}-\rho(\mathbf{v} \cdot \mathbf{n}) \hat{\mathbf{v}} \quad$ on $\quad \Gamma_{\text {out }}$

$\hat{\mathbf{v}} \cdot \mathbf{n}=0, \mathbf{n} \cdot \hat{\boldsymbol{\tau}} \cdot \mathbf{t}=\mathbf{0} \quad$ on $\quad \Gamma_{\text {slip }}$

and with

$\lambda=\hat{p} \mathbf{n}-\mu\left(\nabla \hat{\mathbf{v}}+\nabla \hat{\mathbf{v}}^{T}\right) \cdot \mathbf{n}$

where $\hat{\boldsymbol{\tau}}=\mu\left(\nabla \hat{\mathbf{v}}+\nabla \hat{\mathbf{v}}^{T}\right)-\mathbf{I} \hat{p}$. Together with the PDE constraints of the optimization problem (6b)-(6g) the adjoint equation system (10a)-(10f) characterizes a saddle point of the Lagrangian (7), which is assumed to be a unique stationary point of $\mathcal{L}$. To compute the shape derivative of the augmented Lagrangian (7) the domain $\Omega$ is made variable by a family of transformations $\left\{\mathbf{T}_{t}\right\}_{t \geq 0}$ with the parameterized perturbation of identity

$$
\mathbf{T}_{t}=\mathbf{i d}+t \mathbf{u}_{p}
$$

with $\quad \mathbf{T}_{0}(\Omega)=\Omega$ and $\quad \mathbf{u}_{p} \in V_{0}^{1, p}$,

where $V_{0}^{1, p}:=\left\{\mathbf{u} \in W^{1, p}\left(\Omega, \mathbb{R}^{d}\right): \mathbf{u}=\mathbf{0}\right.$ a.e. on $\left.\partial \Omega \backslash \Gamma\right\}$, and $t \geq 0$ is a step size, cf. Kühl et al. (2019). Application of the first-order optimality condition

$\left.\frac{\partial}{\partial t} \mathcal{L}\left(\mathbf{T}_{t}(\Omega), \mathbf{v}, p, \hat{\mathbf{v}}, \hat{p}, \lambda, \lambda_{b}, \lambda_{c}\right)\right|_{t=0}=0$

leads to the surface formulation of the shape derivative of the objective function $J$, compare e.g., Boisgérault and Zolésio (2000). For its representation, we define

$$
\begin{aligned}
\gamma:= & -\mu \frac{\partial \hat{\mathbf{v}}}{\partial n} \cdot \frac{\partial \mathbf{v}}{\partial n}+\lambda_{b} \cdot \frac{\mathbf{x}-\boldsymbol{\beta}}{\int_{\Omega} 1 \mathrm{~d} x}+\lambda_{c} \\
& +\rho_{b} \frac{(\boldsymbol{\beta}-\overline{\mathbf{b}})(\mathbf{x}-\boldsymbol{\beta})}{\int_{\Omega} 1 \mathrm{~d} x}+\rho_{c}\left(\int_{\Omega} 1 \mathrm{~d} x-\bar{c}\right)
\end{aligned}
$$

where

$\boldsymbol{\beta}=\frac{\int_{\Omega} \mathbf{x} \mathrm{d} x}{\int_{\Omega} 1 \mathrm{~d} x}$ 
is the barycenter of $\Omega$, c.f. Bello et al. (1997); Schulz and Siebenborn (2016). Then

$J^{\prime}(\Omega ; \mathbf{u})=\int_{\Gamma} \gamma \mathbf{u} \cdot \mathbf{n} \mathrm{d} s$,

is the shape derivative of $J$ at $\Omega$ in the direction of $\mathbf{u}$. While the Lagrange multipliers $(\hat{\mathbf{v}}, \hat{p})$ are given by the solution of the boundary value problem (10a) - (10f), the multipliers $\lambda_{b}$ and $\lambda_{c}$ for barycenter and volume constraint are not given by the solution of a system of PDEs and rather have to be determined by an augmented Lagrange method like outlined in algorithm 1 of the next section, c.f. Andreani et al. (2008); Allaire et al. (2021). Hence, with the shape derivative from (15) the minimization problem in (4) with $W^{1, p}\left(\Omega, \mathbb{R}^{d}\right)$ replaced by $V_{0}^{1, p}\left(\Omega, \mathbb{R}^{d}\right)$ is associated with the variational form to the $p$-Laplacian problem

$$
\left.\begin{array}{ll}
-\operatorname{div}\left(\left(\nabla \mathbf{u}_{p}: \nabla \mathbf{u}_{p}\right)^{\frac{p-2}{2}} \nabla \mathbf{u}_{p}\right)=0 & \text { in } \Omega, \\
\left(\nabla \mathbf{u}_{p}: \nabla \mathbf{u}_{p}\right)^{\frac{p-2}{2}} \frac{\partial \mathbf{u}_{p}}{\partial n}=-\gamma \mathbf{n} & \text { on } \Gamma, \\
\mathbf{u}_{p}=0 & \text { on } \partial \Omega \backslash \Gamma .
\end{array}\right\}
$$

Note that $\Gamma \subset \partial \Omega$ is the part of the boundary which is free for deformation. This approach now can also be interpreted as a generalization by the $p$-Laplace setting of the approach proposed in Schulz and Siebenborn (2016) where the descent direction is defined regarding a Steklov-Poincaré operator; in other words the Dirichlet-to-Neumann map is applied to $\gamma \mathbf{n}$. Therewith, the descent direction in Allaire et al. (2004) and Schulz and Siebenborn (2016) is obtained by solving an elliptic boundary value problem similar to

$$
\left.\begin{array}{ll}
-\Delta \mathbf{u}=0 & \text { in } \Omega \\
\frac{\partial \mathbf{u}}{\partial n}=-\gamma \mathbf{n} & \text { on } \Gamma \\
\mathbf{u}=0 & \text { on } \partial \Omega \backslash \Gamma
\end{array}\right\}
$$

which is the Euler-Lagrange equation of the minimization problem

$$
\min _{\mathbf{u} \in V_{0}^{1,2}} \frac{1}{2} \int_{\Omega} \nabla \mathbf{u}: \nabla \mathbf{u} \mathrm{d} x-\int_{\Gamma} \gamma \mathbf{n} \cdot \mathbf{u} \mathrm{d} s,
$$

with $V_{0}^{1,2}=\left\{\mathbf{u} \in H^{1}\left(\Omega, \mathbb{R}^{d}\right): \mathbf{u}=\mathbf{0}\right.$ a.e. on $\left.\partial \Omega \backslash \Gamma\right\}$. This obviously represents a special case of (4) with $p=2$ and serves as a reference for the numerical experiments discussed in Section 4. Let us also note that this concept was enhanced in Onyshkevych and Siebenborn (2021) where a non-linear extension operator has been introduced. The observation of strong distortion of the discrete grid within the main deformation direction motivated adding a nonlinear advection term to the PDE in (17). Therewith, greater deformations are possible and the mesh quality is reasonable even after large deformations of the initial grid. However, as our numerical results show, the $p$-Laplace relaxation of problem (3) provides a systematic approach to fluid dynamic shape optimization also guaranteeing meshes of high quality after large deformations, in particular when the optimal shape has sharp corners.

\section{Optimization algorithm}

In Algorithm 1, we specify our minimization algorithm.

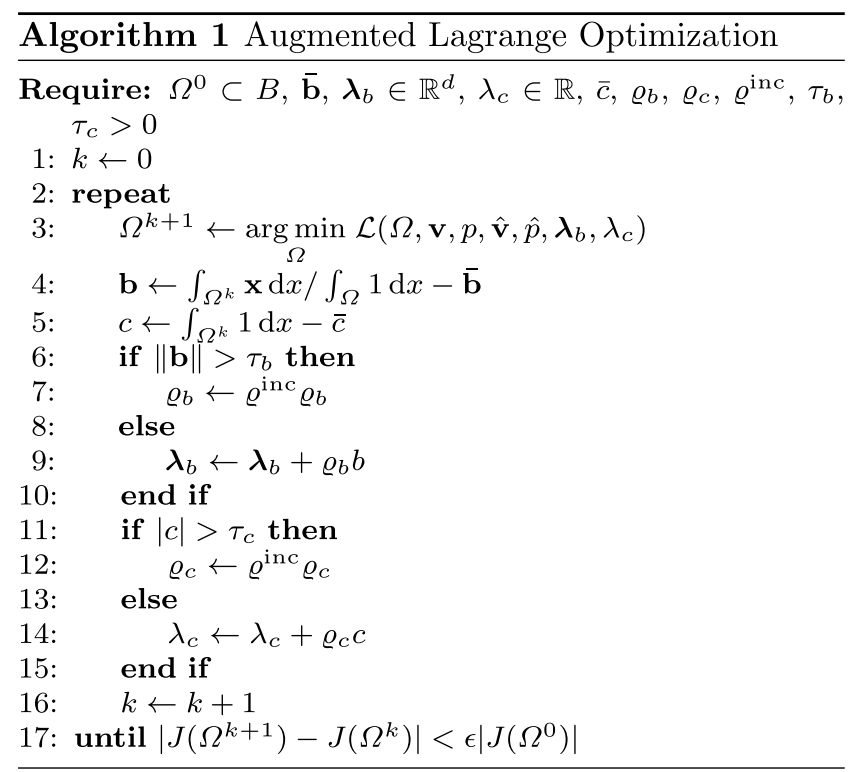

It was used before, in e.g., Schulz and Siebenborn (2016) to determine the multipliers $\lambda_{b}$ and $\lambda_{c}$ for the barycenter and volume constraints of a shape optimization problem. Other than in Schulz and Siebenborn (2016) the update for the penalty factors $\rho_{b}, \rho_{c} \in \mathbb{R}_{+}$and $\lambda_{b} \in \mathbb{R}^{d}$ and $\lambda_{c} \in \mathbb{R}$, respectively, is separated which is motivated by the big difference in the order of magnitude between both penalty factors $\left(\varrho_{b} \propto 10^{8}\right.$ and $\left.\varrho_{c} \propto 10^{2}\right)$ for the numerical experiments in this paper. However, the critical part of algorithm 1 refers to step 3 which is therefore farther explored in algorithm 2 . To compute the multipliers $\lambda_{b}$ and $\lambda_{c}$ Algorithm 2 is performed with decreasing convergence tolerance $\epsilon>0$. This also keeps the computation numerically stable and circumvents unfeasible shapes during the optimization. 


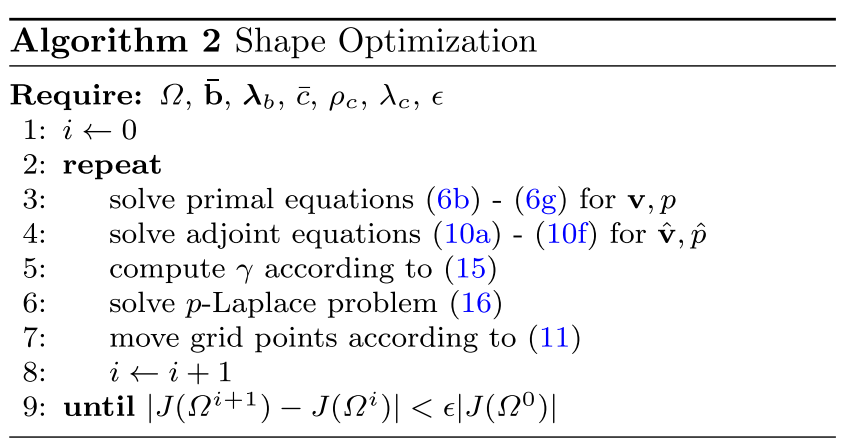

A conventional, pressure-based, second-order accurate finite-volume scheme for a cell-centered variable arrangement is employed to discretize the partial differential equations of the primal (6b)- $(6 \mathrm{~g})$ and adjoint systems (10a)-(10f), cf. Rung et al. (2009), Stück and Rung (2013) and Kühl et al. (2020). The existing infrastructure and generic subroutines of the fluid solver can be re-used with limited effort for the implementation. In case of $p=2$, the initial guess with $\mathbf{u}_{p}=0$ in $\Omega$ leads to convergence of the implementation. To reduce computing time a sequence of problems in $p$ is solved with growing values for $p>2$. We define a finite sequence $\left(p_{1}, \ldots, p_{k}\right)$ with $p_{1}:=2$ and $p_{k}:=p$, and use the solution of the $p_{l}$-Laplacian problem as initialization for the numerical solution process of the $p_{l+1}$-Laplace problem $(0 \leq l<k)$.

\section{Applications}

Three fluid dynamic applications are discussed to investigate the performance of the $p$-Laplace approach (4) in shape optimization. All cases are concerned with drag minimization at steady state and subjected to conserve the wetted volume and its barycenter, c.f. Fig. 1. Starting from the Laplace expression with $p=2$, the first case analyses the influence of increasing $p$ for a frequently referenced $2 D$ Stokes flow example that features a pointed oval optimum. Emphasis is given to (a) the convergence of the optimization, (b) the final shape and the attainable drag reduction as well as (c) the quality of the mesh updates. The second case demonstrates the applicability of the approach for an analog $3 D$ configuration and analyses the same aspects (a-c). Since both initial applications refer to low Reynolds (low Re) number flows, a third example is supplemented to scrutinize the performance in a $2 D$ turbulent flow at high Reynolds number.

\subsection{Drag optimization in 2D low Re flow}

The first case studies the drag minimization of a $2 D$ circular cylinder exposed to low Reynolds number flow for

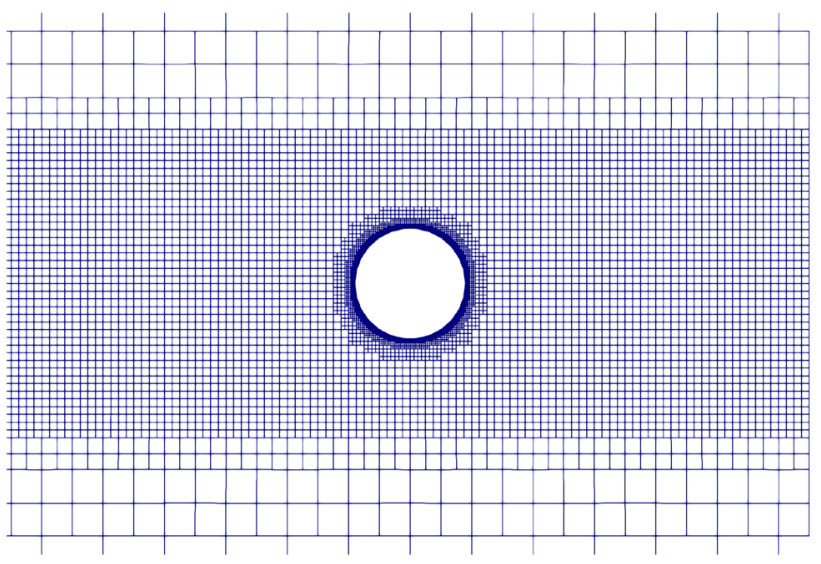

Fig. 2 Initial mesh of the $2 D$ low Reynolds number problem

Table 1 Initial values for the parameters of the augmented Lagrange procedure

\begin{tabular}{llll}
\hline Parameter & $2 D$ low Re & $3 D$ low Re & $2 D$ high Re \\
\hline$\varrho_{b}$ & $5 \cdot 10^{7}$ & $1 \cdot 10^{3}$ & $1.2 \cdot 10^{8}$ \\
$\varrho_{c}$ & $1 \cdot 10^{2}$ & 50 & $4 \cdot 10^{2}$ \\
$\rho^{\text {inc }}$ & 2 & 2 & 1.2 \\
$\tau_{b}$ & $1 \cdot 10^{-6}$ & $1 \cdot 10^{-1}$ & $5 \cdot 10^{-5}$ \\
$\tau_{c}$ & $2 \cdot 10^{-2}$ & $1 \cdot 10^{-4}$ & $2 \cdot 10^{-2}$ \\
$t$ & $2 \cdot 10^{-3}$ & $5 \cdot 10^{-3}$ & $1 \cdot 10^{-3}$ \\
\hline
\end{tabular}

$p=2,3, \ldots, 6$. The computational domain is illustrated in Fig. 1. The case is related to the setting initially described by Pironneau (1973). Instead of the Stokes flow considered in Pironneau (1973), we employ a low Re Navier-Stokes formulation of the boundary value problem.

The initial cylinder features a unit diameter $(D=1[\mathrm{~m}])$ and is centered in a channel of length $50[\mathrm{~m}]$ and height $10[\mathrm{~m}]$. The fluid is characterized by a unit density and dynamic viscosity, i.e., $\rho=1\left[\mathrm{~kg} / \mathrm{m}^{3}\right], \mu=1[P s \cdot s]$. Dirichlet conditions are imposed at the inflow $\Gamma_{\text {in }}$ with $\mathbf{v}_{\infty}=(1,0)^{T}[\mathrm{~m} / \mathrm{s}]$, which yields a unit Reynolds number $R e_{D}=1$. Slip wall boundary conditions are applied to the top and bottom boundaries of the channel. Outflow boundary conditions $(6 \mathrm{~g})$ are used along $\Gamma_{\text {out }}$.

The initial grid is displayed in Fig. 2 and features 927 evenly distributed cells along the circumference of the cylinder boundary $\Gamma$. The CAD-free optimization procedure employs an unstructured, locally refined mesh with approximately $14.5 \mathrm{k}$ control volumes which is deformed along with the surface using (11). Mind that the optimal shape is expected to reveal pointy tips at the front and the aft, which shall deliberately not be preempted by the initial discretization of the boundary $\Gamma$. The target barycentre and wetted volume are set to $\overline{\mathbf{b}}=(0,0)^{T}$ and $\bar{c}=50 \cdot 10-\pi / 4\left[\mathrm{~m}^{2}\right]$ 
respectively. All parameters employed to initialize the algorithms 1 and 2 are displayed in Table 1 . The multipliers for the barycenter and volume are initialized with $\lambda_{b}=(0,0)^{T}$ and $\lambda_{c}=0$, respectively. The sequence of tolerances applied to the convergence criteria of the shape optimisation problem is given by $\epsilon=10^{-1}, 10^{-2}, \ldots, 10^{-7}$. However, it turned out that the multipliers $\lambda_{b}$ and $\lambda_{c}$ converge very fast and after four augmented Lagrange steps the multipliers are determined sufficiently exact in each computation with different values for $p$. The field values for the fixed point iteration for $p=2$ can be initialized with $\mathbf{u}_{p}=0$ for all discrete points. Larger $p$ values were initialized by solutions obtained from the previous smaller value, each to a suitable tolerance to provide an initial guess for the next following $p$-Laplace problem. Although the theory suggests to drive $p \rightarrow \infty$, lower $p$ values are of interest for large-scale applications due to the more exhausting computational effort. The numerical effort for solving the $p$-Laplace problem is investigated in Loisel (2020) for different values for $p$ which show that it is of polynomial complexity but depends on $p$ and the number of unknowns. The related experience from this investigation reveals an increase of computing time $T_{p}$ for one iteration of the shape optimization algorithm 2 by $T_{p=4} / T_{p=3} \approx 2.8$ and $T_{p=5} / T_{p=4} \approx 1$.6. In addition, the representable floating point arithmetic of the machine limits the value for $p$.

\subsubsection{Optimal shapes and convergence}

Figure 3 shows the normalized evolution of the drag objective (8) over a selected number of optimization steps for the five investigated values of $p$. The baseline solution refers to $p=2$ which is also related to the investigations in Schulz and Siebenborn (2016).

Table 2 outlines a comparison of performance indicators obtained for the five investigated values of $p$. The last column refers to the maximum number of design steps that are needed to reach a sufficiently converging objective function (cf. algorithm 2). The table reveals that the
Table 2 Performance indicators obtained with different $p$ values for 2D low Re case

\begin{tabular}{lllll}
\hline$p$ & Tip angle $\left[{ }^{\circ}\right]$ & $a / b_{(i t=50)}$ & $J / J^{0}$ & conv. step \\
\hline 2 & 163.8 & 1.4 & 0.9243 & $/$ \\
3 & 152.4 & 1.6 & 0.9222 & 415 \\
4 & 140.4 & 1.8 & 0.9211 & 337 \\
5 & 129.4 & 2.0 & 0.9202 & 278 \\
6 & 116.4 & 2.1 & 0.9195 & 239 \\
\hline
\end{tabular}

convergence improves and fewer optimization steps are needed for larger values of $p$. For $p=2$ the optimization could not reach convergence, but terminated after 356 steps due to grid quality issues, which are discussed below. At this step, the convergence criterion was about two orders of magnitude above the threshold. However, from a practical point of view the convergence criteria employed in this study might appear rather strict and practical applications would also reach sufficient optima for $p=2$. Table 2 also displays the final objective function values, which again reveal improvements for increasing $p$ values. Drag reductions refer to about $7.6 \%$ for the Laplacian approach with $p=2$ and increase to approximately $8.1 \%$ for $p=6$. Improvements seen for the objective function are attributed to the more extreme deformations obtained from the $p$-Laplacian problem (4) with $p>2$. To judge the final shape, the opening angle at the upstream tip may also serve as a measure. The interior opening angles listed in Tab. 2 decrease with greater values of $p$. Hence, increasing $p$ clearly yields more pointy tips as also indicated by the comparison of tip shapes in Fig. 4. The Stokes flow problem investigated in Pironneau (1973) reported an opening angle of $120^{\circ}$. The present results rapidly approach the reported opening angle from above. However, for $p=6$ the opening angle falls below the reference value, which is attributed to the use of Navier-Stokes instead of the Stokes flow model Pironneau (1973). When attention is
Fig. 3 Influence of $p$ value on the evolution of the normalized objective functional (2D low Re case)

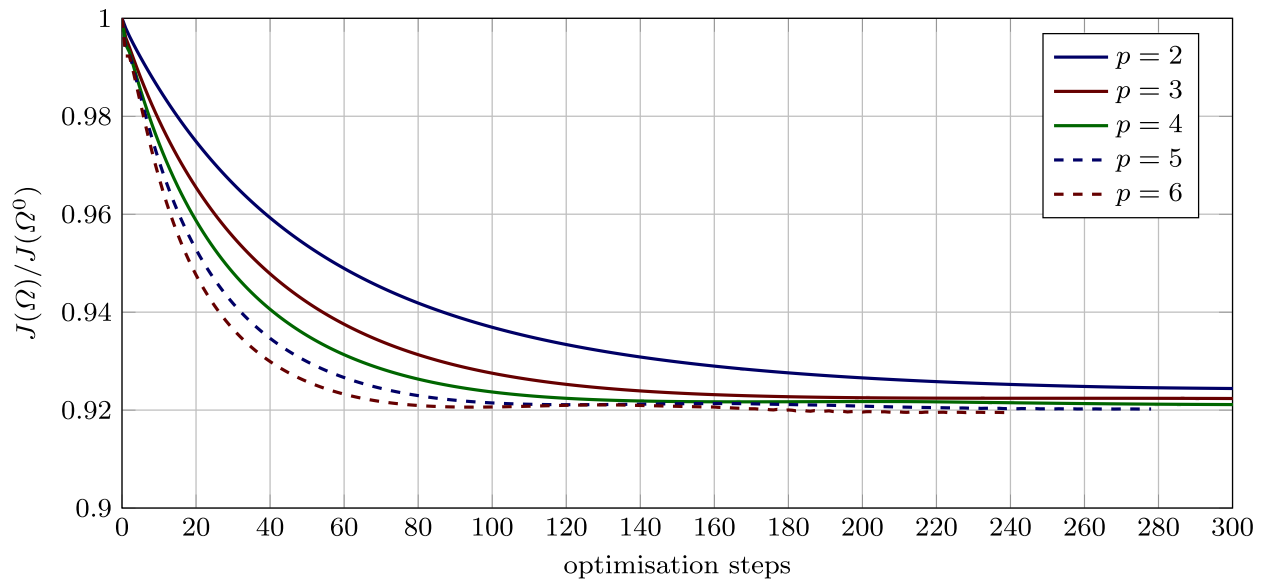


Fig. 4 Influence of the $p$ value on the predicted optimal (final) shapes for the 2D low Re case

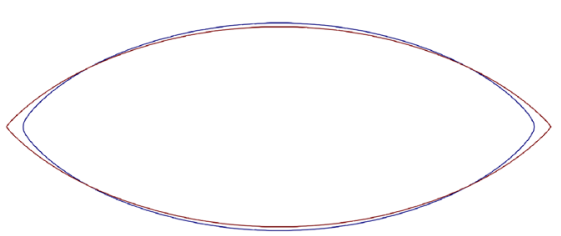

(a) $p=2$ (blue) and $p=3$ (red).

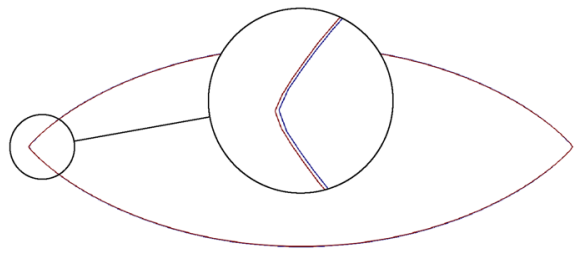

(c) $p=4$ (blue) and $p=5$ (red).

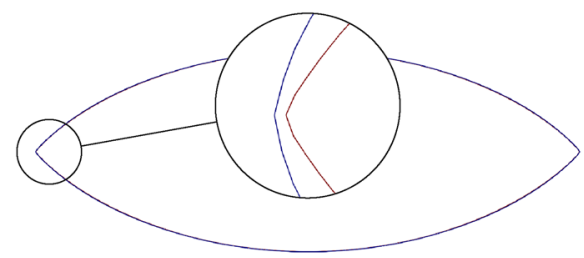

(b) $p=3$ (blue) and $p=4$ (red).

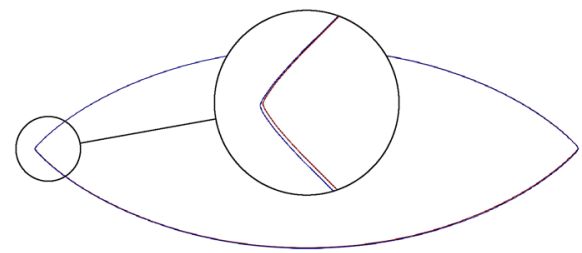

(d) $p=5$ (blue) and $p=6$ (red). directed to the convergence speed, the half axis ratio at the 50th design step, i.e., $a / b_{(i t=50)}$, mentioned in Tab. 2 may be considered as a measure to assess the convergence speed. As all simulations start with $a / b=1$, the tabulated data renders the influence of $p$ values on the ability of the $p$-Laplace approach to rapidly adjust the shape. It is also observed, that large deformations take place at an early stage of the optimization. For example, the final half axis ratio for $p=4$ refers to approximately 2.7 and already $2 / 3$ of this ratio are reached after 50 design steps. A closer inspection of Fig. 3 reveals, that the value of the objective function slightly increases after a quick descent for $p=5$ (step 120-160) and $p=6$ (step 90-140). This is possible due to the absence of a step size control and occurs because the multipliers that control the barycenter and the displacement strictly speaking only hold for a preceding iteration. Moreover, the $p$-Laplace problem (4) is not solved exactly in every iteration of algorithm 2 and preceding results are used as the initial guess for a subsequent optimization step. A similar phenomenon can be seen in results reported by Allaire et al. Allaire et al. (2004), who did use a similar augmented Lagrange procedure.

The contours of the final shapes are depicted in Fig. 4, where optimal shapes for two consecutive values of $p$ are compared with each other, i.e., for $p=2$ with $p=3, p=3$ with $p=4$ and so forth. The shape contours in Fig. 4a show significant differences, in particular at the tips of the resulting geometry. The computation with $p=2$ clearly leads to a more rounded shape and a larger vertical extent than the other investigated $p$ values. Shapes returned by $3 \leq p \leq 6$ are displayed in Fig. 4b-d. Remarkably, a general difference between the respective contours is hard to identify for $p \geq 3$. Thus, close-ups are used to assess the tip region. While a rounded tip region is still observed for $p=3$, the tip becomes more pointy for $p \geq 4$ Figure $4 \mathrm{c}$, d only displays small differences between the shapes obtained with $p=4,5,6$. Thus, one can assume that the predicted optimal shapes converge with $p$ large enough, e.g., $p \geq 4$.

To estimate the convergence behavior in $p$, we measure the symmetric difference between the optimized shapes obtained for $p \geq 2$ and the initial circular shape. To this end, we use the mapping $\mathrm{d}: \mathcal{A} \times \mathcal{A} \rightarrow \mathbb{R}$, with $\mathcal{A}$ as in Sect. 2, defined by

$\mathrm{d}\left(\Omega_{p}, \Omega_{q}\right):=\int_{\mathbb{R}^{d}} \chi_{p, q}(\mathbf{x}) \mathrm{d} x$

with $\Omega_{p}, \Omega_{q} \in \mathcal{A}$ and the indicator function

$\chi_{p, q}(\mathbf{x}):= \begin{cases}1 & : \mathbf{x} \in\left(\Omega_{p} \backslash \Omega_{q}\right) \cup\left(\Omega_{q} \backslash \Omega_{p}\right), \\ 0 & : \text { else. }\end{cases}$

Figure 6 illustrates the region in which the indicator function (20) is not zero.

Figure 7 shows the difference, in the sense of (19), between the initial and the optimal shapes obtained for $p \in[2,6]$. It is seen, that the symmetric difference between the initial and the optimal shape seems to converge with increasing $p \geq 3$. This observation shall not be taken as a proof but may show the relation of our approach to the investigations in Ishii and Loreti (2005).

Note that the reference case $p=2$ is referred to as a Hilbert space method and does not belong to $\left\{\mathbf{u}_{p}\right\}_{p>2}$ with $\mathbf{u}_{p} \in W^{1, p}\left(\Omega, \mathbb{R}^{d}\right)$. Therefore, we assessed the transition regime between $p=2$ and $p=3$ in greater detail. As indicated by Fig. 7, an increase of $p$ above the reference value $p=2$ results in a rapid response of the symmetric difference between $\Omega_{p}$ and $\Omega_{0}$ 


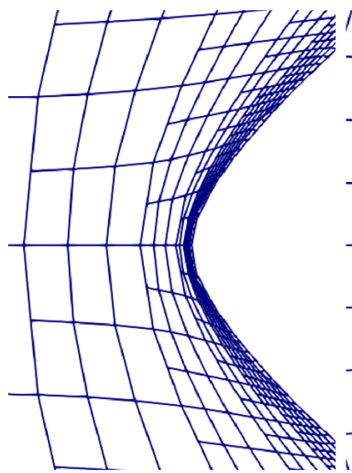

(a) $p=2$

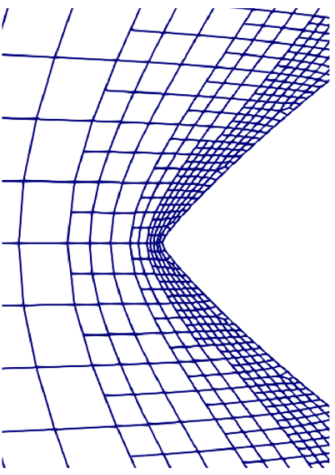

(b) $p=3$

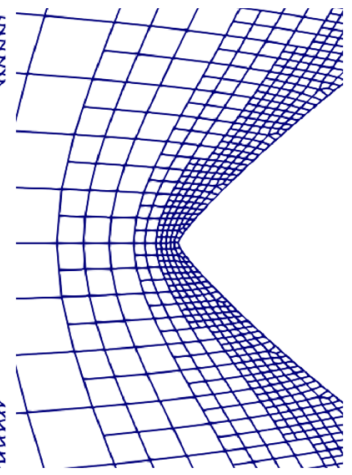

(c) $p=4$

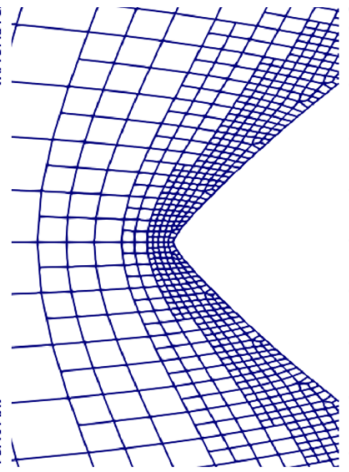

(d) $p=5$

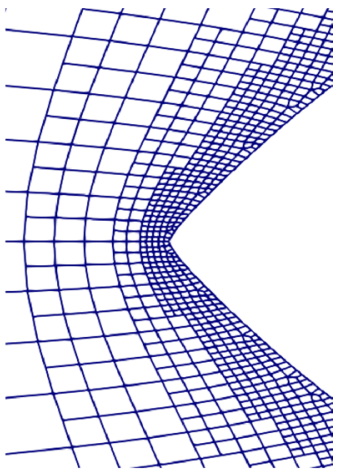

(e) $p=6$

Fig. 5 Influence of the $p$ value on the final grids in the vicinity of the upstream tip (2D low Re case)

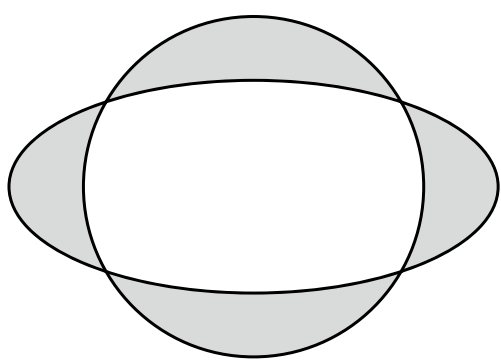

Fig. 6 Illustration of the symmetric difference between an optimized shape $\Omega_{p}$ and the initial shape $\Omega_{0}$

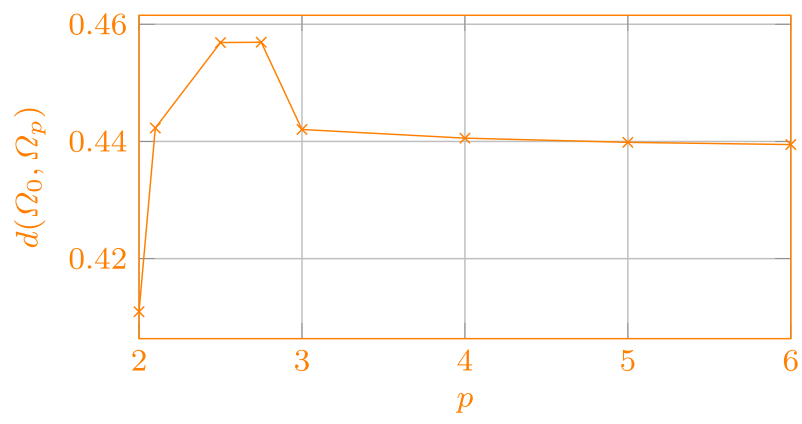

Fig. 7 Evolution of the symmetric difference between the optimized shape $\Omega_{p}$ and the initial shape $\Omega_{0}$ for the $2 \mathrm{D}$ low Re number case

\subsubsection{Grid deformation}

Besides the convergence, the attainable optimal shapes and the reduction of the objective function value, another major aspect refers to the quality of the mesh updates. Maintaining the grid quality during the optimization process is crucial to the success of the CAD-free optimization procedure. In this study, we focus on (a) the grid orthogonality near the boundaries as well as (b) the cell aspect ratio in the vicinity

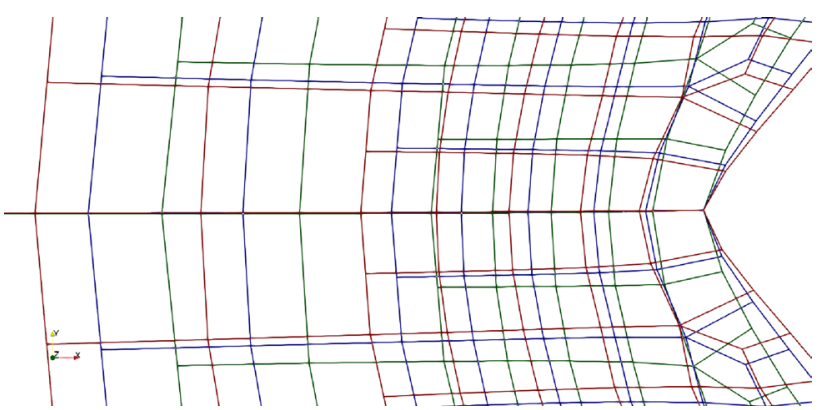

Fig. 8 Superposition of upstream tip grids for $p=4$ (green), $p=5$ (blue) and $p=6$ (red) for the 2D low Re case

of the walls. It is seen, that using the $p$-Laplacian problem (4) with $p>2$ significantly improves the quality of the mesh updates in comparison with updates obtained from $p=2$. Fig. 5a-e displays the final grids in the upstream tip region for the five investigated values of $p$. A reasonable grid quality is generally observed for large values of $p$, even after substantial cumulative deformations due to several hundred optimization steps. On the contrary, the aspect ratio of the near-wall cells in Fig. 5a increases significantly for $p=2$. The cells become stretched and tend to buckle in normal direction, which hampers the iterative convergence of the primal and adjoint flow solver. Therefore the procedure terminated after 354 optimization steps for $p=2$. In line with the change of the shape characteristics, a huge change of the mesh characteristics is observed when $p$ is increased from $p=2$ to $p=3$, cf. Fig. 5a, b. The grid is less compressed in the vicinity of the upstream tip for $p=3$, despite the larger deviations from the initial grid indicated in Fig. 4a. As outlined by Fig. $5 \mathrm{c}-\mathrm{e}$, the grid does further improve when $p$ is augmented to $p=4,5,6$.

A detailed comparison of the grids at the upstream tip of the final shape follows from Fig. 8 for $p=4$ (green), $p=5$ (blue) and for $p=6$ (red). The post processed grids are 
Fig. 9 Cell aspect ratio of the final shapes obtained with $p=2$ (left) and $p=3$ (right) for the 2D low Re case
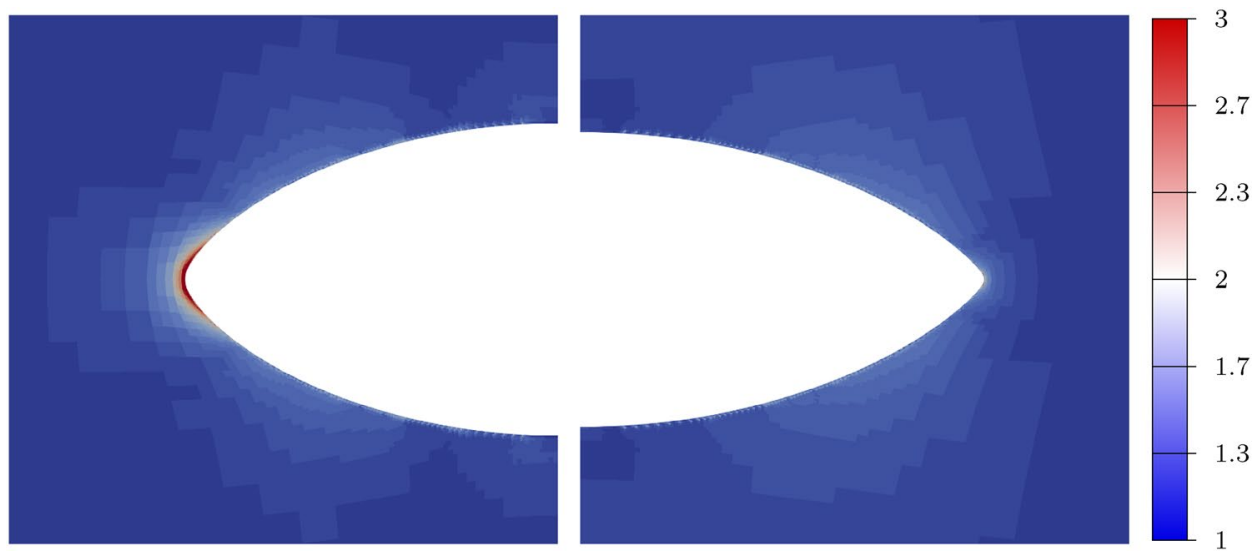

Fig. 10 Minimum interior cell angle for the final meshes obtained with $p=2$ (left) and $p=3$ (right) for the 2D low Re case
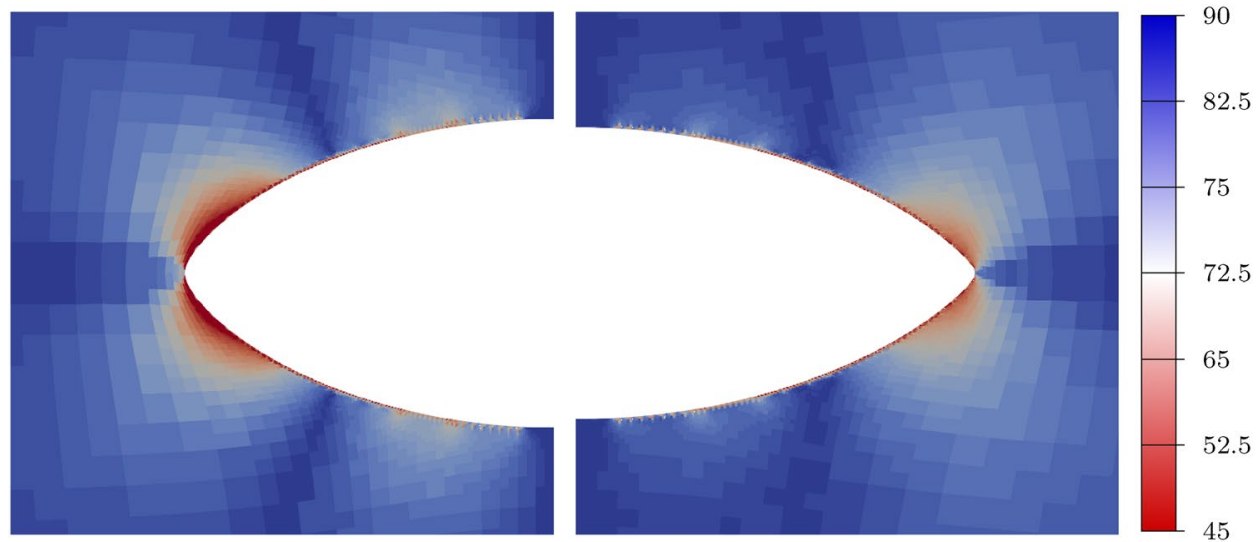

aligned at the tip to support the comparison. The stretching of the grid increases with increasing $p$, which becomes obvious when observing the spacing along the horizontal center line.

Figure 9 depicts contour plots of the cell aspect ratio for the final grids obtained with $p=2$ (left) and $p=3$ (right). These $p$ values are particularly illustrative, since they denote a threshold for the characteristics of the mesh and the shape. The aspect ratio (AR) of the initial grid is generally close to $A R=1$. Only cells within the first two layers next to the cylinder boundary initially reach aspect ratios of up to $A R \leq 1.5$. If one focuses on the final meshes, computations using $p=2$ lead to a substantial amount of cells where the aspect ratio exceeds values of $A R=2.5$ and beyond, particularly in the vicinity of the tip. The maximum values approximately read $A R_{\text {max }} \leq 11$. A significant improvement is achieved in conjunction with $p=3$. Here, only few cells of the final mesh, located within a small area around the upstream tip, display aspect ratios greater than $A R>1.5$ and the peak values of the aspect ratio are limited to $A R_{\text {max }} \leq 3$.

The orthogonality of the cells within a mesh is of particular interest for the approximation of the boundary layer flow around the obstacle. Figure 10 displays contour plots for the minimum interior angle of the final meshes using
Table 3 Performance indicators obtained with different $p$ values for the 3D low Re case

\begin{tabular}{lllll}
\hline$p$ & Tip angle $\left[{ }^{\circ}\right]$ & $\frac{1}{2}\left(\frac{a}{b}+\frac{a}{c}\right)_{(o p t)}$ & $J / J^{0}$ & conv. step \\
\hline 2 & 176.4 & 1.61 & 0.9507 & $/$ \\
4 & 130.2 & 2.13 & 0.9360 & 164 \\
\hline
\end{tabular}

$p=2$ (left) and $p=3$ (right). Displayed angles reach from a less favorable value of $45^{\circ}$ (red) to a preferred value of $90^{\circ}$ (blue). Skewed cells occur close to tip and in regions where the shape tends toward a straight edge. This effect is much more pronounced for $p=2$ (left) and significantly less obvious for $p=3$ (right). As illustrated by Fig. 8, the characteristic features of the mesh do not substantially change for higher $p$ values.

\subsection{Drag optimization in 3D low Re flow}

This subsection is devoted to the drag optimization of a $3 D$ unit-diameter sphere exposed to low Reynolds number flow. Reported results are limited to the baseline case $p=2$ and a single augmented $p$-level of $p=4-$ which did already display substantial benefits in the $2 D$ study. The computational 
Fig. 11 Drag optimization of a sphere at $R e=1$; final shape obtained from $p=2$ after 94 steps

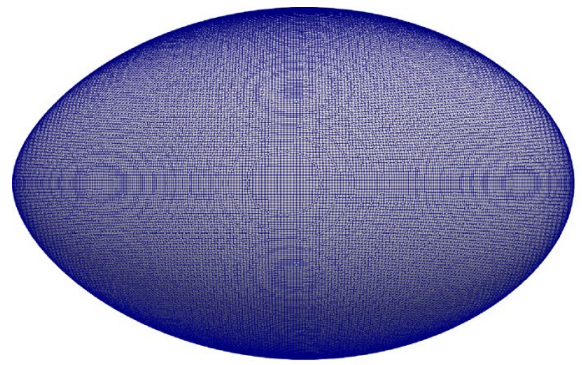

(a) Side view.

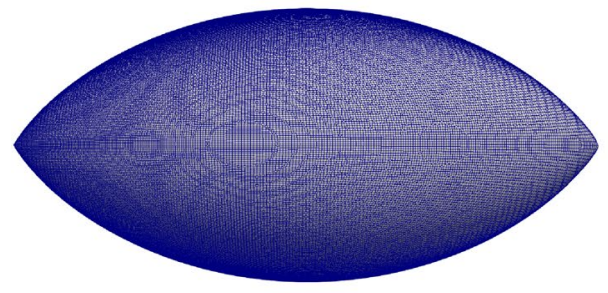

(a) Side view

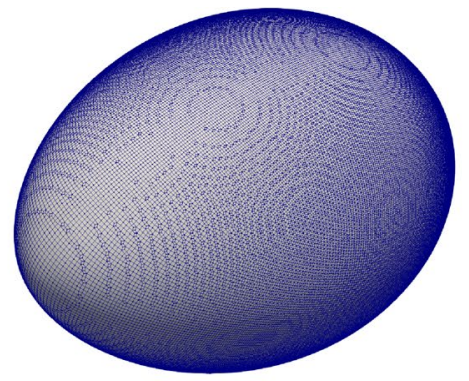

(b) Perspective view onto the upstream tip.

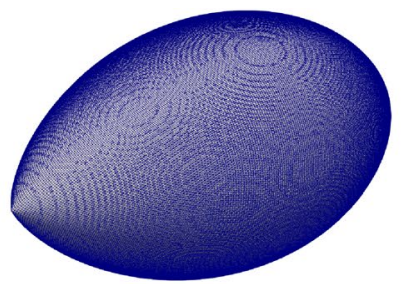

(b) Perspective view onto the upstream tip.

domain, the position of the obstacle's barycenter and the boundary conditions agree with the $2 D$ case outlined in Sect. 4.1. The $2 D$ domain is supplemented in lateral direction by $10[\mathrm{~m}]$, and slip wall conditions are imposed along the lateral boundaries. The Reynolds number compiled with the diameter and the approaching flow again reads $R e=1$.

The boundary $\Gamma$ of the sphere is resolved by approximately $113 \mathrm{k}$ wall adjacent cells and deforms under control. The total grid features about $1050 \mathrm{k}$ control volumes. The wetted volume is restricted to conserve $\bar{c}=5000-\pi / 6\left[\mathrm{~m}^{3}\right]$ and the initial parameters of the augmented Lagrange algorithm 1 are again denoted in Table 1 . The performance observed with the two investigated $p$ values is summarized in Table 3. For $p=4$ the optimization did converge after 164 design steps and yields $6.4 \%$ drag reduction. Similar to the $2 \mathrm{D}$ case, the lower quality of the volume grid update restricted the amount design steps for $p=2$. Figure 11 depicts the last shape obtained from computations with $p=2$ which referred to 94 design steps and $4.93 \%$ drag reduction. Fig. 11a reveals that this shape is clearly characterized by round tips at the upstream and downstream ends.

The optimal shape returned by $p=4$ is shown in Fig. 12 . As also indicated by the data listed in Table 3, pointy upstream and downstream ends are seen for $p=4$ which also results in a significantly larger average half axis ratio.

\subsection{Drag optimization in 2D turbulent flow}

Supplementary to the two low Reynolds number cases described above, we report the results obtained for a turbulent $2 D$ drag optimization of an initial $a / b=4 / 1$ ellipses

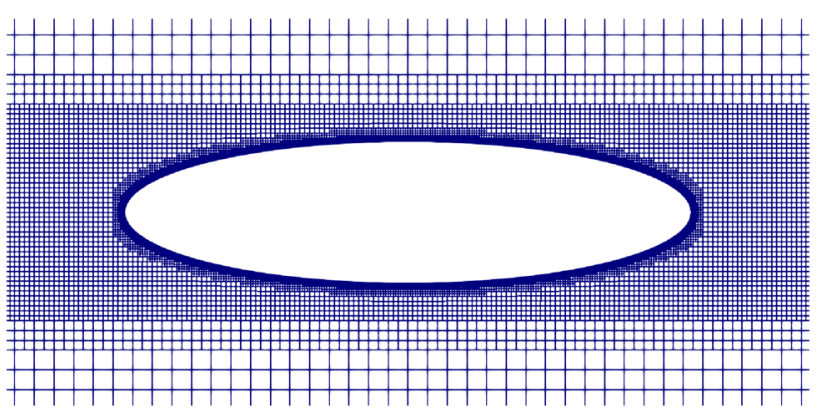

Fig. 13 Initial mesh for the drag optimization of a $2 D$ ellipses exposed to horizontal approach flow at $R e=3 \cdot 10^{6}$

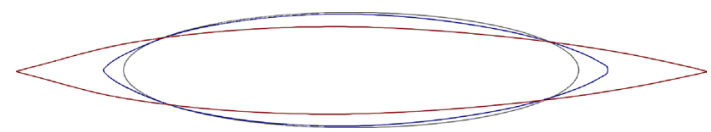

Fig. 14 Drag optimization of a $2 D$ elliptical cylinder exposed to horizontal approach flow at $R e=3 \cdot 10^{6}$; Comparison of the final shapes obtained for $p=2$ (blue) and $p=4$ (red) against the initial shape (gray)

where the longer half axis has a unit length of $a=1[\mathrm{~m}]$. The incompressible fluid is characterized by a unit density and a dynamic viscosity of $\mu=6 . \overline{66} \cdot 10^{-7}[\mathrm{~Pa} \cdot \mathrm{s}]$. The governing equations refer to RANS equations using a standard $k-\omega$ eddy-viscosity turbulence model Wilcox (1998) in combination with a wall-function approach. The Reynolds number based on the longer axis of the ellipses and the approach flow reads $R e=3 \cdot 10^{6}$. The computational domain and the 
Table 4 Performance indicators obtained with different $p$ values for the $2 D$ high Re case

\begin{tabular}{lllll}
\hline$p$ & Tip angle $\left[{ }^{\circ}\right]$ & $a / b_{(o p t)}$ & $J / J^{0}$ & conv. step \\
\hline 2 & 149.5 & 4.55 & 0.894 & $/$ \\
4 & 29.4 & 8.02 & 0.674 & 589 \\
\hline
\end{tabular}

boundary conditions agree with the information already used in the first example, cf. Sect. 4.1. Results are again restricted to the baseline case $p=2$ and $p=4$. The wetted volume and the target barycenter are identical to those applied to the low Reynolds number experiment in Sect. 4.1. The initial values for the algorithim 1 are denoted within the third column of Table 1.

The initial grid again features local grid refinement near the boundary of the design surface to ensure an adequate resolution of the high curvature region and is depicted in Fig. 13. The design surface $\Gamma$ is discretized by approximately 2300 cells of equal size and the volume grid features $46 \mathrm{k}$ cells.

The final shape contours obtained for $p=2$ (blue) and $p=4$ (red) are outlined in Fig. 14. Data listed in Table 4 reveal a significant difference of the shape and the drag reduction experienced with the different $p$ values. Similar to the $3 D$ study, pointy upstream and downstream ends are seen for $p=4$. The larger $p$ value results in a significant increase of the initial half axis ratio, while $a / b$ hardly increases for the smaller $p$ value. As in the previous studies, the shape optimization terminated before an optimal shape could be reached due to the severe distortion of the grid for the baseline value $p=2$. For the baseline approach $p=2$, the drag force of the body is reduced by $10.57 \%$ within 540 optimization steps. However, for $p=4$ the drag force is reduced by $32.6 \%$ when reaching the convergence criterion.

Figure 15 compares the final grids in the vicinity of the upstream tip for $p=2$ (left) and $p=4$ (right). As indicated by Fig. $15 \mathrm{a}$, the aspect ratio deteriorates for $p=2$ since the near-wall cells stretch in tangential direction. Moreover, cells (again) cluster at the tip. In contrast, the grid for $p=4$ depicted in Fig. 15b features evenly distributed cells along the design surface. The predicted shape displays a pointy upstream tip which is a much better approximation to the solution to the optimization problem.

A similar conclusion follows from Fig. 16 which describes the situation at the downstream end. It is observed that, the pointy ends develop at later stages of the optimization process, particularly in the rear. Thus, the (almost) pointy rear is hardly reached for $p=2$. In combination with $p=4$, the cell distribution at the rear remains evenly

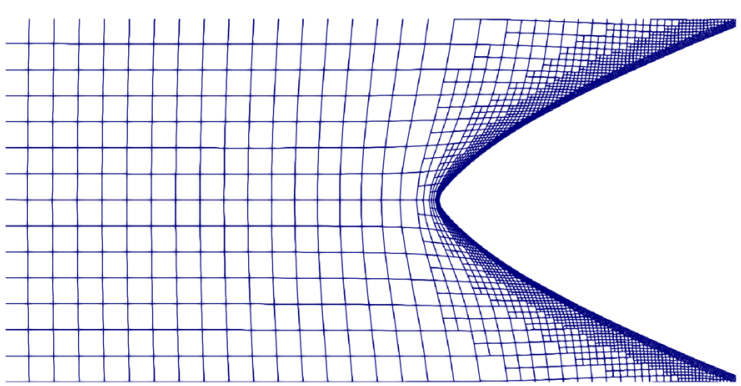

(a) $p=2$

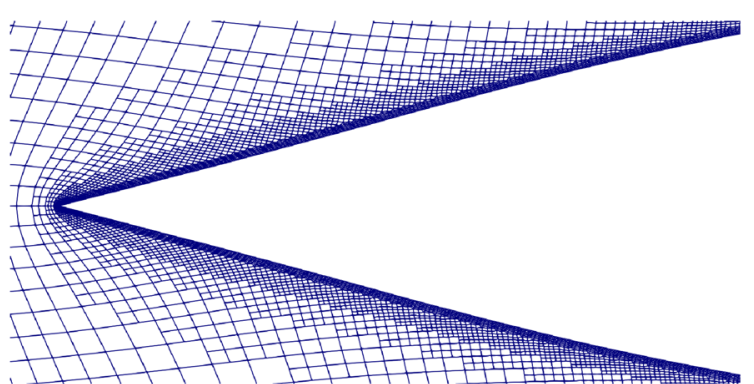

(b) $p=4$

Fig. 15 Upstream tip of final shapes for the $2 D$ high Reynolds case

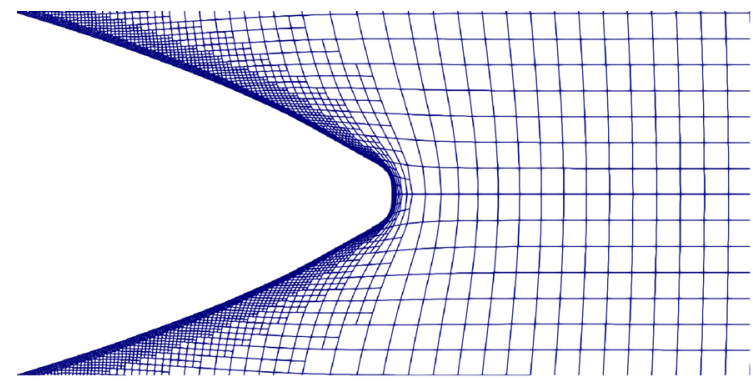

(a) $p=2$

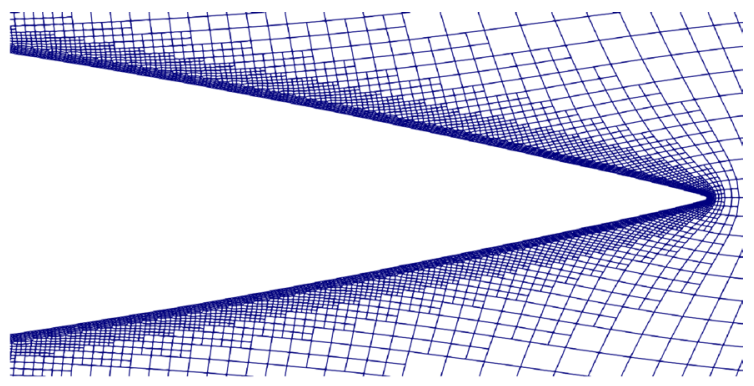

(b) $p=4$

Fig. 16 Downstream tip of final shapes for the 2D high Reynolds case 
distributed. Mind that a small round tip can still observed in Fig. 16b. To receive a sharp pointy tip at the downstream end, a step size control would be beneficial.

\section{Conclusions}

We presented a novel approach for shape optimization by approximating Lipschitz continuous transformations based on the relaxation of the definition of the steepest descent direction. Examples included were restricted to fluid dynamic applications, but also apply to other simulation areas. The main goal was to improve the shape optimization algorithm by considering a descent direction as the solution to the $p$-Laplace problem, and investigate the influence of increasing $p$ on the convergence of the shape optimization procedure as well as the obtained shapes and the related updates of the volume grid. An important aspect refers to the behavior of the relative differences when $p$ is increased, since this might guide toward sufficiently high $p$ values associated with appreciated lower computational effort.

Results show that directions obtained from $p$-harmonic solutions improve the convergence with increasing $p$. At the same time, the optimal shapes improve regarding the value of the objective function. A remarkable feature is related to the ability of the $p$-Laplace approach to yield shapes with edges or pointy shapes, even when the initial shape does not contain such features. Furthermore, the quality of the computational grid is virtually preserved even when large deformations of the initial shape occur and no specific grid adjustment is required. Results of the present study suggest that $p=4$ seems a sufficiently large $p$ value to gather the benefits of the $p$-harmonic approach.

Within future research different solution algorithms for the $p$-Laplace problem may be considered to improve the approximation of the steepest descent direction and thus reduce the computational efforts. Moreover, applications to large-scale 3D problems may be investigated.

Acknowledgements The authors acknowledge the support by the Deutsche Forschungsgemeinschaft (DFG) within the Research Training Group GRK 2583 "Modeling, Simulation and Optimization of Fluid Dynamic Applications" as well as within the research project " Drag Optimisation of Ship Shapes" (Grant No. RU 1575/3-1). Michael Hinze acknowledges support of the DFG Priority Programme 1962 with projekt P8 "A Non-Smooth Phase-Field Approach to Shape Optimization with Instationary Fluid Flow". Selected computations were performed with resources provided by the North-German Super-computing Alliance (HLRN).

Author contributions PMM: Conceptualization, Methodology, Software, Validation, Formal analysis, Investigation, Writing-original draft, Writing — review \& editing, Visualization. NK: Software, Validation, Formal analysis, Investigation, Writing - original draft, Writing-review \& editing. MS: Funding acquisition, Conceptualization, Methodology, Writing — review \& editing. KD: Idea, mathematical consulting. MH: Idea, mathematical consulting, methodology, Writing-review \& editing. TR: Project administration, Funding acquisition, Supervision, Conceptualization, Methodology, Resources, Writing-original draft, Writing — review \& editing.

Funding Open Access funding enabled and organized by Projekt DEAL.

\section{Declarations}

Conflict of interest The authors declare that they have no conflict of interest.

Replication of results All geometries for the studied test cases are described analytically. For numerical computations a proprietary mesh generation software was used.

Open Access This article is licensed under a Creative Commons Attribution 4.0 International License, which permits use, sharing, adaptation, distribution and reproduction in any medium or format, as long as you give appropriate credit to the original author(s) and the source, provide a link to the Creative Commons licence, and indicate if changes were made. The images or other third party material in this article are included in the article's Creative Commons licence, unless indicated otherwise in a credit line to the material. If material is not included in the article's Creative Commons licence and your intended use is not permitted by statutory regulation or exceeds the permitted use, you will need to obtain permission directly from the copyright holder. To view a copy of this licence, visit http://creativecommons.org/licenses/by/4.0/.

\section{References}

Allaire G, Dapogny C, Jouve F (2021) Chapter 1-shape and topology optimization. In: Geometric partial differential equations-Part II, vol 22. Handbook of numerical analysis. Elsevier, New York, pp 1-132. https://doi.org/10.1016/bs.hna.2020.10.004

Allaire G, Jouve F, Toader A-M (2004) Structural optimization using sensitivity analysis and a level-set method. J Comput Phys 194(1):363-393. https://doi.org/10.1016/j.jcp.2003.09.032

Andreani R, Birgin EG, Martínez JM, Schuverdt ML (2008) On augmented Lagrangian methods with general lower-level constraints. SIAM J Optim 18(4):1286-1309. https://doi.org/10.1137/06065 4797

Azegami H, Fukumoto S, Aoyama T (2013) Shape optimization of continua using NURBS as basis functions. Struct Multidiscip Optim 47(2):247-258. https://doi.org/10.1007/s00158-012-0822-4

Azegami H, Wu ZC (1996) "Domain Optimization Analysis in Linear Elastic Problems: Approach Using Traction Method". In: JSME International Journal 39.2, pp. 272-278. https://doi.org/10.1299/ jsmea1993.39.2_272

Bello JA, Fernández-Cara E, Lemoine J, Simon J (1997) The differentiability of the drag with respect to the variations of a Lipschitz domain in a Navier-Stokes flow. SIAM J Control Optim 35(2):626-640. https://doi.org/10.1137/S0363012994278213

Bletzinger K-U (2014) A consistent frame for sensitivity filtering and the vertex assigned morphing of optimal shape. Struct Multidiscip Optim 49(6):873-895. https://doi.org/10.1007/s00158-013-1031-5

Boisgérault S, Zolésio JP (2000) Shape derivative of sharp functionals governed by Navier-Stokes flow. In: Jäger W, Necas J, John O, Najzar K, Stará J (eds) Partial differential equations: theory and numerical solution, pp 49-63. 
Borrval, T, Petersson J (2003) Topology optimization of uids in Stokes flow. Int J Numer. Methods Fluids 41:77-107. https://doi.org/10. $1002 / \mathrm{fld} .426$

Deckelnick K, Herbert P, Hinze M (2021) A novel W1;1 approach to shape optimisation with Lipschitz domains. arXiv: 2103.13857

Delfour MC, Zolésio J -P (2011) Shapes and geometries: metrics, analysis, differential calculus, and optimization. SIAM. https:// doi.org/10.1137/1.9780898719826

Dwight R, Brézillon J (2006) Effects of various approximations of the discrete adjoint on gradient- based optimization. In: AIAA paper 2006690

Garcke H, Hecht C, Hinze M, Kahle C (2015) Numerical approximation of phase field based shape and topology optimization for uids. SIAM J Sci Comput 37:A1846-A1871. https://doi.org/10.1137/ 140969269

Garcke H, Hecht C, Hinze M, Kahle C, Lam A (2016) Shape optimization for surface functionals in Navier-Stokes flow using a phase field approach. Interfaces Free Bound 18:219-261.

Garcke H, Hinze M, Kahle C, Lam KF (2018) A phase field approach to shape optimization in Navier-Stokes ow with integral state constraints. Adv Comput Math 44:1345-1383. https://doi.org/ 10.1007/s10444-018-9586-8

Haubner J, Siebenborn M, Ulbrich M (2020) A continuous perspective on modeling of shape optimal design problems. arXiv: 2004. 06942

Hicks RM, Henne PA (1978) Wing design by numerical optimization. J Aircraft 15(7):407-412. https://doi.org/10.2514/3.58379

Hinze M, Pinnau R, Ulbrich M, Ulbrich S (2008) Optimization with PDE constraints, vol 23. Springer, Berlin. https://doi.org/10.1007/ 978-1-4020-8839-1

Ishii H, Loreti P (2005) Limits of solutions of p- Laplace equations as $\mathrm{p}$ goes to infinity and related variational problems. SIAM J Math Anal 37(2):411-437. https://doi.org/10.1137/S003614100 4432827

Jameson A, Vassberg JC (2000) Studies of alternative numerical optimization methods applied to the brachistochrone problem. Int $\mathbf{J}$ Comput Fluid Dyn 9(3):281-296

Kawohl B, Pironneau O, Tartar L, Zolésio J-P (1998) Optimal shape design. Springer, Berlin

Kröger J, Rung T (2015) CAD-free hydrodynamic optimisation using consistent kernel-based sensitivity filtering. Ship Technol Res 62(3):111-130. https://doi.org/10.1080/09377255.2015.1109872

Kühl N, Kröger J, Siebenborn M, Hinze M, Rung T (2020) Adjoint complement to the volume of-fluid method for immiscible flows. arXiv: 2009.03957

Kühl N, Müller PM, Stück A, Hinze M, Rung T (2019) Decoupling of control and force objective in adjoint-based fluid dynamic shape optimization. AIAA J 57(9):4110-4114. https://doi.org/10.2514/1. J058376

Löhner R, Soto O, Yang C (2003) An adjointbased design methodology for CFD optimization problems. In: 41st aerospace sciences meeting and exhibit. p 299. https://doi.org/10.2514/6.2003-299

Loisel S (2020) Efficient algorithms for solving the p- Laplacian in polynomial time. Numerische Mathematik 146(2):369-400. https:// doi.org/10.1007/s00211-020-01141-z

Onyshkevych S, Siebenborn M (2021) Mesh quality preserving shape optimization using nonlinear extension operators. J Optim Theory Appl. https://doi.org/10.1007/s10957-021-01837-8
Othmer C (2008) A continuous adjoint formulation for the computation of topological and surface sensitivities of ducted flows. Int $\mathrm{J}$ Numer Methods Fluids 58(8):861-877. https://doi.org/10.1002/ fld. 1770

Othmer C (2014) Adjoint methods for car aerodynamics. J Math Ind 4(1):6. https://doi.org/10.1186/2190-5983-4-6

Papoutsis-Kiachagias EM, Giannakoglou KC (2016) Continuous adjoint methods for turbulent flows, applied to shape and topology optimization: industrial applications. Archiv Comput Methods Eng 23(2):255. https://doi.org/10.1007/s11831-014-9141-9

Pironneau O (1973) On optimum profiles in stokes flow. J Fluid Mech 59(1):117-128. https://doi.org/10.1017/S002211207300145X

Robinson TT, Armstrong CG, Chua HS, Othmer C, Grahs T (2012) Optimizing parameterized CAD geometries using sensitivities based on adjoint functions. Comput Aided Des Appl 9(3):253268. https://doi.org/10.3722/cadaps.2012.253-268

Rung T, Wöckner K, Manzke M, Brunswig J, Ulrich C, Stück A (2009) Challenges and perspectives for maritime CFD applications. Jahrbuch der Schiffbautechnischen Gesellschaft 103:127-39

Schmidt S, Ilic C, Schulz V, Gauger NR (2013) Three-dimensional large-scale aerodynamic shape optimization based on shape calculus. AIAA J 51(11):2615-2627. https://doi.org/10.2514/1.J052245

Schulz V, Siebenborn M (2016) Computational comparison of surface metrics for PDE constrained shape optimization. Comput Methods Appl Math 16(3)::485-496. https://doi.org/10.1515/ cmam-2016-0009

Sokolowski J, Zolésio J-P (1992) Introduction to shape optimization. Shape sensitivity analysis. Springer, Berlin

Soto O, Löhner R, Yang C (2002) A stabilized pseudo-shell approach for surface parametrization in CFD design problems. Commun Numer Methods Eng 18(4):251-258. https://doi.org/10.1002/ cnm. 490

Soto O, Löhner R, Yang C (2004) An adjoint-based design methodology for CFD problems. Int J Numer Methods Heat Fluid Flow 14(6):734-759. https://doi.org/10.1108/09615530410544292

Stavropoulou E, Hojjat M, Bletzinger K-U (2014) In-plane mesh regularization for node-based shape optimization problems. Comput Methods Appl Mech Eng 275:39-54. https://doi.org/10.1016/j. cma.2014.02.013

Stück A, Rung T (2013) Adjoint complement to viscous finite-volume pressure-correction methods. J Comput Phys 248:402-419. https://doi.org/10.1016/j.jcp.2013.01.002

Stück A, Rung T (2011) Adjoint RANS with filtered shape derivatives for hydrodynamic optimisation. Comput Fluids 47(1): 22-32. https://doi.org/10.1016/j.compfluid.2011.01.041

Ulbrich M, Ulbrich S (2012) Nichtlineare optimierung. Springer, Berlin

Vassberg J, Jameson A (2006) Aerodynamic shape optimization part 1: theoretical background. In: Introduction to optimization and multidisciplinary design, pp. 1-30

Vassberg J, Jameson A (2006) Aerodynamic shape optimization part 2: sample applications. In: Introduction to optimization and multidisciplinary design, pp 1-41

Wilcox DC (1998) Turbulence modeling for CFD, vol. 2. DCW industries La Canada, CA

Publisher's Note Springer Nature remains neutral with regard to jurisdictional claims in published maps and institutional affiliations. 